Yadira Alexandra Calle-Bermeo; Darwin Gabriel García-Herrera; Sandra Elizabeth Mena-Clerque; Juan Carlos Erazo-Álvarez

http://dx.doi.org/10.35381/e.k.v3i1.1019

\title{
Aprendizaje basado en problemas y trabajo colaborativo para la enseñanza de Matemática
}

\section{Problem-based learning and collaborative work for the teaching of Mathematics}

\author{
Yadira Alexandra Calle-Bermeo \\ yadira.calle.92@est.ucacue.edu.ec \\ Universidad Católica de Cuenca, Azogues \\ Ecuador \\ https://orcid.org/0000-0001-5785-1971 \\ Darwin Gabriel García-Herrera \\ dggarciah@ucacue.edu.ec \\ Universidad Católica de Cuenca, Azogues \\ Ecuador \\ https://orcid.org/0000-0001-6813-8100 \\ Sandra Elizabeth Mena-Clerque \\ sandramena@ucacue.edu.ec \\ Universidad Católica de Cuenca, Cuenca \\ Ecuador \\ https://orcid.org/0000-0002-9186-2161 \\ Juan Carlos Erazo-Álvarez \\ jcerazo@ucacue.edu.ec \\ Universidad Católica de Cuenca, Cuenca \\ Ecuador \\ https://orcid.org/0000-0001-6480-2270
}

Recepción: 10 agosto 2020

Revisado: 25 septiembre 2020

Aprobación: 15 octubre 2020

Publicación: 01 noviembre 2020 


\title{
RESUMEN
}

La investigación tuvo como objetivo analizar el aprendizaje basado en problemas y trabajo colaborativo para la enseñanza de Matemática en la Escuela de Educación Básica "Joel Monroy" ubicada en la ciudad de Cuenca, parroquia Baños, sector Misicata. Metodológicamente fue de carácter descriptivo correlacional no experimental transversal. Respecto a los estudiantes encuestados se puede observar que el valor de sig. para el Chi cuadrado de bilateral Pearson es menor que 0.05 demostrando que la variable es paramétrica asumiendo que la hipótesis es afirmativa (H1). Es por ello que el (ABP) aprendizaje basado en problemas a través del trabajo colaborativo genera en los estudiantes curiosidad, motivación e interés por aprender dentro de las clases. facilitando la adquisición e interpretación de un problema matemático.

Descriptores: Aprendizaje activo; método de aprendizaje; matemáticas. (Palabras tomadas del Tesauro UNESCO).

\begin{abstract}
The objective of the research was to analyze problem-based learning and collaborative work for the teaching of Mathematics at the "Joel Monroy" Basic Education School located in the city of Cuenca, Baños parish, Misicata sector. Methodologically, it was descriptive, correlational, non-experimental, cross-sectional. Regarding the surveyed students, it can be observed that the value of sig. for the two-sided Pearson Chi-square it is less than 0.05 showing that the variable is parametric assuming that the hypothesis is affirmative $(\mathrm{H} 1)$. That is why $(\mathrm{ABP})$ problem-based learning through collaborative work generates in students curiosity, motivation and interest in learning within classes. facilitating the acquisition and interpretation of a mathematical problem.
\end{abstract}

Descriptors: Activity learning; learning methods; mathematics. (Words taken from the UNESCO Thesaurus). 
Yadira Alexandra Calle-Bermeo; Darwin Gabriel García-Herrera; Sandra Elizabeth Mena-Clerque; Juan Carlos Erazo-Álvarez

\section{INTRODUCCIÓN}

Hoy en día vivimos en una sociedad de cambios radicales y acelerados con los avances tecnológicos, sociales y más aún educativos, lo que permite que los conocimientos, herramientas y estrategias metodológicas vayan cambiando tanto en el aprendizaje como en la enseñanza de las matemáticas, (Caballero-Jiménez \& Espínola-Reyna, 2016), indican que el miedo a las matemáticas es en general debido a malas experiencias que tuvieron en sus calificaciones o reprobaron un año, por lo que reciben amonestaciones tanto de los padres como del docente influyendo negativamente y creando un ambiente tenso y hostil. En ciertos casos el primer día de clases el docente parte de lo que piensa que los estudiantes deben saber y no desde sus experiencias o su conocimiento, por ende, se crea el rechazo a la materia llegando a una frustración y un rechazo definitivo. Al respecto se busca soluciones para mejorar la enseñanza-aprendizaje de la matemática lo cual se ha ido analizando en varios países, uno de ellos fue en los Congresos Iberoamericanos CIBEM realizado en la ciudad de Montevideo-Uruguay, también en la ciudad de Matanza Cuba evento llamado MATECOMPU sobre temas del ¿Cómo?, ¿Cuándo? y ¿Por qué? enseñar y aprender Matemática, en Bogotá-Colombia en el año 2016 IBERCIENCIA con el tema: "Enseñando matemáticas explicando que el aprender no es cuestión de entrada de información si no de comprensión es decir por el desarrollo del pensamiento matemático". Entre otros temas y la búsqueda de soluciones que preocupan al docente por enseñar y guiar al estudiante y dar posibles soluciones para mejorar el desarrollo lógico matemático.

Con lo mencionado anteriormente, cabe recalcar que el docente es una parte clave en la educación, ya que con sus ideas innovadoras pueden mejorar el uso de las herramientas pedagógicas, además de tener un pensamiento crítico y analítico que contribuye a fortalecer el desarrollo lógico en el estudiante para poder llegar a un aprendizaje significativo, que les permita afianzar y profundizar dichos conocimientos para su aplicación diaria. 
Si bien es cierto una de las mayores dificultades que presenta el Ecuador es el área de matemática demostrando un desinterés, apatía a la materia, desmotivación y miedo dando como resultado el bajo rendimiento académico, por ello la necesidad de buscar técnicas y metodologías activas que requieran un aprendizaje activo que nos ayude a establecer encadenamientos lógicos de razonamiento. Con los resultados que obtuvo el Ecuador en las pruebas PISA-D 2018, nos ha hecho reflexionar la forma de cómo estamos impartiendo las clases de matemáticas si lo hacemos solo de manera mecánica o estamos realmente activando un pensamiento crítico.

El Ecuador participó por primera vez en la capacidad de resolver problemas matemáticos, dando como resultado un 70,9\% de dificultad alcanzando el nivel 2 en matemática ubicándose en el nivel de desempeño básico, con un promedio de 377 sobre 1000, esto hace pensar mucho como docentes y que si no se tienen bases académicas, ni conocimientos adecuados, destrezas y habilidades en las matemáticas sea en cualquier nivel básico, los estudiantes pasarán a la básica superior y al bachillerato con deficiencia en los conocimientos. (Schleicher, 2016) menciona que PISA realiza evaluaciones en el área de matemática y lengua en donde analizan la capacidad de razonamiento, procedimientos y conceptos matemáticos de la misma forma analizan la capacidad de ver el mundo matemático para una cooperación activa en esta sociedad actual.

La investigación tuvo como objetivo analizar el aprendizaje basado en problemas y trabajo colaborativo para la enseñanza de Matemática en la Escuela de Educación Básica "Joel Monroy" ubicada en la ciudad de Cuenca, parroquia Baños, sector Misicata. 
Yadira Alexandra Calle-Bermeo; Darwin Gabriel García-Herrera; Sandra Elizabeth Mena-Clerque; Juan Carlos Erazo-Álvarez

\section{Referencial teórico}

\section{Problemas de aprendizaje en matemática}

En los últimos años por la preocupación del bajo rendimiento en dicha asignatura varios grupos académicos se han dedicado en investigar la problemática de la enseñanza aprendizaje del área de matemáticas tan etiquetado y odiado por la mayoría de los estudiantes; con el fin de proponer y aplicar mejores alternativas y estrategias didácticas para solucionar este problema, en este sentido, (Terán-de-Serrentino \& Pachano-Rivera, 2005), indica que desde el punto de vista tradicional las clases de matemáticas empiezan comunmente con definiciones formales con terminos dificiles de entender, y continuan con varios problemas matemáticos lejos de su contexto, llevando a los estudiantes a varias incognitas sin saber cual es realmente el objetivo de aprender matemáticas quedando la preocupacion en el docente de como llegar al estudiante con los conocimientos necesarios en su aprendizaje.

Tenemos que considerar que los modelos y estrategias de enseñanza aprendizaje utlizadas en clases por el docente tiene mucho que ver con el aprendizaje del estudiante, cambiar el hacer, enseñar y comunicar las matemáticas de una manera práctica y no mecánica, es la que obliga al docente a estar a la vanguardia en la educación buscando mejores alternativas sea mediante capacitaciones y actualización de conocimientos pedagógicos para implementar en las aulas de clase de manera innovadora y óptima para nuestros estudiantes para asi fortalecer las competencias matemáticas que nos exige en el curriculum nacional en la sociedad actualidad.

Si tomamos como referencia a posiciones epistemológicas contructivistas de Vygotsky sobre la interaccion social y el aprendizaje colaborativo fundamental e indispensable en el crecimiento de un desarrollo cognitivo y con la mano del aprendizaje significativo de David Ausubel, estamos dejando de lado un aprendizaje memorístico, mecánico que mantiene la educación tradicional y partimos a un aprendizaje activo en donde despierte el interés del estudiante y la creatividad por aprender. (Gibert \& Ballester, 2013) menciona 
que los procesos de matemática debe ser contextualizados a su realidad tomando en consideracion el desarrollo del pensamiento lógico matemàtico de los estudiantes de una manera interactiva despertando un pensamiento crítico en la resolución de problemas de la vida cotidiana, sin dejar de lado la parte integral de su personalidad con la ayuda del docente mediador con sus conocimientos sólidos.

Por otro lado, (Moreno-Pinado \& Velázquez-Tejeda, 2017), en su artículo "Estrategia Didàctica para desarrollar el pensamiento Crítico" hace un aaportan en la contribuciòn de mejorar el desarrollo del pensamiento crítico en los estudiantes de Quinto año de secundaria de la Institucion educativa "San Mateo de Huanchor" en donde menciona que la falta de asimilación y comprensión de orden teórico y didáctico de parte de los docentes que no utilizan estrategias problematizadoras y metacognitivas que exista reflexión, comprensiòn y criticidad en el desarrollo del estudiante; los estudiantes demuestran deficiencia tanto en conocimientos y habilidades del pensamiento impidiendo a un análisis, comprensión e interpretación limitando a un desarrrollo del pensamiento y proponiendo una metodología que estimule la capacidad y habilidades del pensamiento con una didáctica desarrolladora de manera interdisciplinaria con metodologias activas.

Así mismo, (Bartau-Rojas, et al., 2017) De acuerdo a su Investigacion sobre "metodología de enseñanza en centros eficaces de la comunidad de Autonomía del País Vasco demuestra en los centros educativos un reaizado en trabajo en grupo de manera colaborativa, utilizaron el aprendizaje basado en problemas y proyectos entre otros y otro grupo de centros educativos que utilizan las TICs como recurso didàctico, dio resultados positivos en el desarrollo profesional de los docentes como estudiantes esta metodologia lo han venido utilizando un buen tiempo y han ido mejorando y adecuando las estrategias metodologicas activas en base a las necesidades del estudiante los que ha dado eficacia en los centros educativos Integrando lo aprendido y lo realizado. 
En otra investigación (Leiva, 2016), propone en este proyecto de investigacion "ABP como estratégia para desarrollar el pensamiento lógico matemático en alumnos de educación secundaria" propone mejorar habilidades matemàticas a través de estrategias que puedan alcanzar el nivel IV que pide PISA, proponiendo varios modelos explícitos para la resolución de problemas complejos, destacando que el docente es parte escencial para una educacion de calidad dejando de lado el tradicionalismo y el temor a la materia, por lo que menciona al ABP como una estrategia que ayuda a la reflexión, aptitudes sociales y efectivas, pero sobre todo habilidades para un auto aprendizaje.

En este sentido, (Perez \& Ramirez, 2011) menciona que los docentes deben manejar bien la materia con una pedagogía correcta, para que puedan concatenar conocimientos teóricos y prácticos, conocer bien las etapas de la resolución de problemas y contextualizarlos a la realidad del estudiante de manera creativa y original que represente esfuerzos cognoscitivos para que puedan llegar a un razonamiento. En base a ellos el docente debe ser un mediador que afiance sus conocimientos creando en ellos la motivación por la materia y destrezas de acuerdo a su nivel de desarrollo que le permitan retención y transferencia de conocimientos de una manera constructivista promoviendo habilidades cognitivas.

\section{Aprendizaje Basado en Problemas (ABP)}

Si tenemos claro que en nuestro país tenemos un currículo abierto y flexible que se basa en varias concepciones teóricas y metodologías considerando uno de ellos la pedagógica critica en donde el centro del aprendizaje es el estudiante de manera activa e interdisciplinaria siendo las matemáticas unas de las materias indispensables para el desarrollo de la vida en donde le ayuda a desarrollar capacidades para analizar, interpretar y resolver problemas basados a su entorno, fundamentándose en lineamientos del Ministerio de Educación a través de estándares básicos. De acuerdo a lo que menciona (Ministerio de Educacion del Ecuador 2019): 
Yadira Alexandra Calle-Bermeo; Darwin Gabriel García-Herrera; Sandra Elizabeth Mena-Clerque; Juan Carlos Erazo-Álvarez

La enseñanza de la Matemática tiene como propósito fundamental desarrollar la capacidad para pensar, razonar, comunicar, aplicar y valorar las relaciones entre las ideas y los fenómenos reales. Este conocimiento y dominio de los procesos le dará la capacidad al estudiante para describir, estudiar, modificar y asumir el control de su ambiente físico e ideológico, mientras desarrolla su capacidad de pensamiento y de acción de una manera efectiva (p. 362)

Si bien es cierto el área de matemáticas es indispensable para resolver problemas de la vida cotidiana, el docente debe estar siempre preparado para ofrecer las herramientas necesarias de manera óptima al estudiante y adquiera destrezas significativas con estrategias metodológicas adecuadas de manera activa para que la clase se vuelva interactiva dando las oportunidades necesarias a todos los estudiantes de acuerdo a sus capacidades.

Con respecto a los niveles de Educación Básica la enseñanza de las matemáticas se vuelve complicada no solo porque deben enseñar los docentes a los estudiantes a desarrollar operaciones básicas, si no que el estudiante debe tener criticidad en identificar analizar, ordenar e interpretar, cualquier problema que se le presente; buscando las mejores estrategias para que puedan desarrollar un pensamiento matemático.

En complemento, (Kyeong-Ha, 2003) dice que el Aprendizaje basado en problemas aplicado dentro de las clases de matemáticas son más cuestionadas que las clases tradicionales puesto que el trabajo del docente es insertar al estudiante como centro de estudio, más allá de presentar los contenidos matemáticos debe fortalecer la comprensión y desarrollo del razonamiento de manera eficaz con una variedad de problemas matemáticos.

\section{El aprendizaje basado en problemas con el trabajo Colaborativo.}

Así también (Chagas, 2011) menciona que el ABP proporciona al docente ser un mediador del aprendizaje para aumentar habilidades significativas a través de la 
Yadira Alexandra Calle-Bermeo; Darwin Gabriel García-Herrera; Sandra Elizabeth Mena-Clerque; Juan Carlos Erazo-Álvarez

cooperación y la colaboración de grupo en la resolución de problemas. Si se tiene claro que para un aprendizaje activo se debe analizar variedad de técnicas y metodologías, (Lira, 2010) menciona que la metodología activa son estrategias que facilitan al estudiante a desenvolverse en un rol activo dentro del proceso de enseñanza aprendizaje, dentro de una sociedad participativa, permitiendo de esta manera una comunicación asertiva y colaborativa.

Cabe mencionar que existe una variedad de estrategias metodológicas que deben ser aplicadas para el desarrollo de un pensamiento crítico en el área de matemática y uno de ellos es el Aprendizaje basado en problemas mediante un trabajo en equipo (TraviesoValdés \& Ortiz-Cárdenas), al respecto dice que el Aprendizaje basado en problemas $(A B P)$, le mantiene al alumno de una manera activa en la enseñanza ya que despierta la curiosidad por aprender, la creatividad para desarrollar diversas actividades y afianza el desarrollo crítico realizando un compendio de lo teórico y práctico desarrollando un autodidactismo por aprender y la capacidad de autoevaluarse.

Si analizamos desde el puno de vista pedagógico se menciona a Jon Dewey conocido como el padre de la educación renovada en donde sostiene que el estudiante no es sujeto pasivo si no activo en la educación, por lo que podemos relacionar que la metodología activa nace de la pedagogía crítica no solo de John Dewey sino de Freire, Carl Rogger entro otros. Así mismo, (Matamorros, 2018) dice que el Aprendizaje basado en problemas se basa en las teorías del aprendizaje constructivista en donde permite al alumno a ser parte activa del aprendizaje basado en contextos reales del estudiante para que puedan ser resueltos teniendo acceso a la información y material necesario y así obtener varias alternativas de respuestas y saber escoger la correcta.

De lo anteriormente expuesto el Aprendizaje basado en problemas permite desarrollar competencias para la resolución de problemas matemáticos ayudando al desarrollo integral del estudiante de manera competitiva partiendo de un problema que puede ser contextualizado a la realidad para identificar la necesidad de aprendizaje desarrollando 
Yadira Alexandra Calle-Bermeo; Darwin Gabriel García-Herrera; Sandra Elizabeth Mena-Clerque; Juan Carlos Erazo-Álvarez

aptitudes de aspecto lógico, para ello se trabaja conjuntamente con el trabajo colaborativo siendo los dos que coinciden que el estudiante es el centro de su aprendizaje. Trabajan en pequeños y grandes grupos, se les asigna roles, trabajan mediante una comunicación asertiva y discusión para llegar a acuerdos en búsqueda de varias alternativas de solución y al final la evaluación, que promueve destrezas necesarias, mejora la capacidad de aprendizaje y la crítica de habilidades.

En complemento, (Adolphus, et al., 2013) aporta que en el trabajo colaborativo el estudiante es el protagonista de su propio aprendizaje identificando donde está el problema y analiza su solución a través de varias alternativas trabajadas en grupo para la resolución de problemas, así mismo, (Fernandez \& Fonseca, 2016) menciona algunas ventajas del Aprendizaje Basado en problemas:

- Estudiantes motivados en el aprendizaje

- Aprendizaje significativo identificando problemas relevantes en el contexto

- Fortalece el desarrollo de las habilidades del pensamiento de manera eficaz y creativo, nace una cultura de autoevaluación.

- Evita el memorismo y retiene información para construir nuevos aprendizajes en base a problemas planteados.

- Iniciativa de un trabajo autónomo en búsqueda de información.

- Trabajo en equipo permitiendo compartir o defender sus puntos de vista. 
Yadira Alexandra Calle-Bermeo; Darwin Gabriel García-Herrera; Sandra Elizabeth Mena-Clerque;

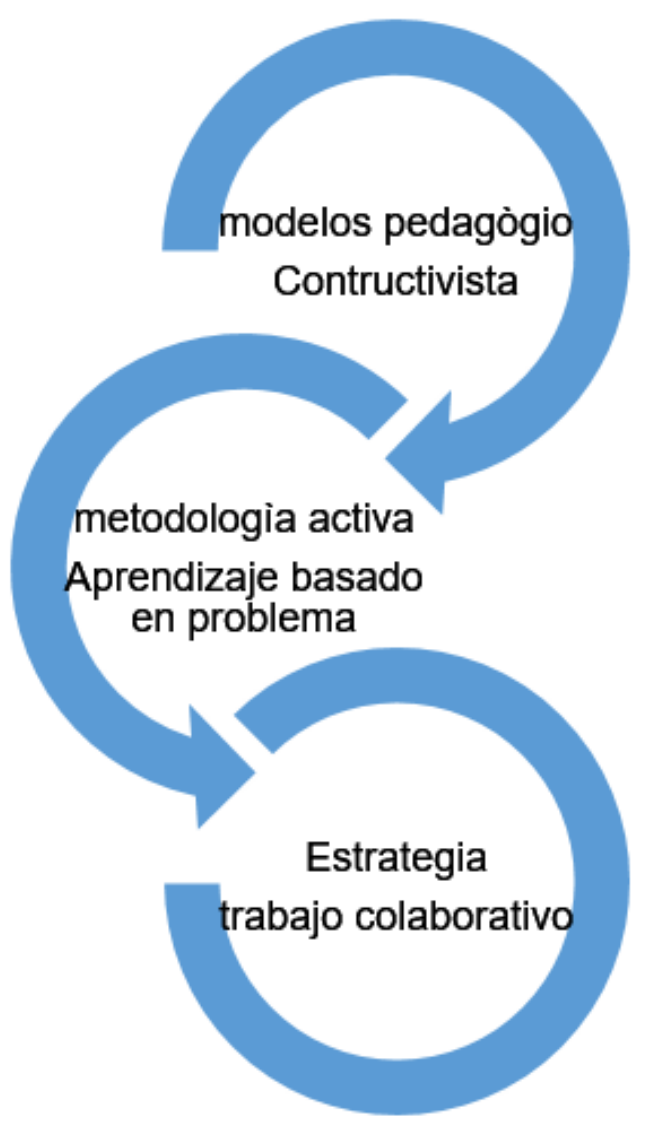

Figura 1. Combinación del modelo pedagógico, metodología activa y trabajo colaborativo.

Fuente: Elaboración propia.

Finalmente se puede acotar que con la implementación de la metodología del Aprendizaje Basado en Problemas (ABP) apoyado en el trabajo colaborativo se puede llegar a un trabajo autónomo con una evaluación constructiva del estudiante que puede ser de manera individual o grupal de manera crítica con una interacción social de cultura y costumbres aprendiendo con responsabilidad y criticidad dando o no veracidad a lo que leen y aprenden desarrollando un pensamiento crítico. 
Yadira Alexandra Calle-Bermeo; Darwin Gabriel García-Herrera; Sandra Elizabeth Mena-Clerque; Juan Carlos Erazo-Álvarez

\section{METODOLOGÍA}

El presente trabajo de investigación sobre el aprendizaje basado en problemas y el trabajo colaborativo: como estrategia metodológica para la enseñanza de matemática en educación general básica fue de carácter descriptivo correlacional no experimental transversal, (Guanipa, 2011) dice que las personas que realizan investigaciones con tendencias cuantitativas se basan en transformar las observaciones en parte numérica siguiendo una seriación.

El estudio se realizó en la Escuela de Educación Básica "Joel Monroy" ubicada en la ciudad de Cuenca, parroquia Baños, sector Misicata, el universo poblacional fue de 12 docentes del área de matemáticas y 244 estudiantes de la institución. Se recolectó los datos a través de una encuesta y cuestionario tanto a los docentes como a los estudiantes, de 13 preguntas en escala de Likert que fueron validadas con el empleo de Software SPSS, con un coeficiente de Alfa de Cronbach de 0.858 de fiabilidad. La información fue analizada mediante estadistica descriptiva y chi cuadrado de Pearson.

\section{RESULTADOS}

Luego de ser analizados los resultados de las encuestas, se puede comprobar que todas las variables obedecen a una distribución normal paramétrica por ello la recolección y análisis es cuantitativo con Chi cuadrado tanto para docentes como para estudiantes, a diferencia que para los docentes se utilizó Shapiro Wilk ya que los encuestados fueron 12 docentes en el área de matemática y para los estudiantes se aplica Kolmogorov puesto que se encuesto a 244 estudiantes.

También se realiza un análisis de frecuencias de una variable de los docentes teniendo los siguientes resultados: 
Yadira Alexandra Calle-Bermeo; Darwin Gabriel García-Herrera; Sandra Elizabeth Mena-Clerque; Juan Carlos Erazo-Álvarez

\section{Tabla 1.}

Como considera usted el nivel de razonamiento lógico matemático de los estudiantes de la Institución.

\begin{tabular}{cccccc}
\hline & & Frecuencia & Porcentaje & Porcentaje válido & $\begin{array}{c}\text { Porcentaje } \\
\text { acumulado }\end{array}$ \\
\hline \multirow{4}{*}{ Válidos } & Muy buena & 2 & 16,7 & 16,7 & 16,7 \\
& Buena & 9 & 75,0 & 75,0 & 91,7 \\
& Regular & 1 & 8,3 & 8,3 & 100,0 \\
\cline { 2 - 6 } & Total & 12 & 100,0 & 100,0 & \\
\hline
\end{tabular}

Fuente: Elaboración propia.

En base a estos indicadores estadísticos de la tabla 1, se observa que el $16.70 \%$ de los docentes consideran que el nivel de razonamiento lógico matemático de los alumnos es muy buena, mientras que el $83.30 \%$ manifiestan que este razonamiento oscila entre bueno y regular de acuerdo a la escala de Likert, lo que nos lleva a suponer que se podría mejorar el razonamiento lógico matemático en los alumnos con la implementación de nuevas estrategias metodológicas como es el Aprendizaje basado en problemas (ABP) con ayuda del trabajo colaborativo. 
Yadira Alexandra Calle-Bermeo; Darwin Gabriel García-Herrera; Sandra Elizabeth Mena-Clerque;

\section{Tabla 2.}

Utiliza estrategias metodológicas activas en sus clases. * A utilizado el Aprendizaje basado en problemas (ABP) como estrategia metodológica en el desarrollo de sus clases.

\begin{tabular}{|c|c|c|c|c|c|c|}
\hline & & \multicolumn{4}{|c|}{$\begin{array}{l}\text { A utilizado el Aprendizaje basado } \\
\text { en problemas (ABP) como } \\
\text { estrategia metodológica en el } \\
\text { desarrollo de sus clases. }\end{array}$} & \multirow{2}{*}{ Total } \\
\hline & & \multicolumn{2}{|c|}{ A veces } & \multicolumn{2}{|r|}{ Casi siempre } & \\
\hline Utiliza estrategias & A veces & \multicolumn{2}{|r|}{2} & \multicolumn{2}{|r|}{0} & 2 \\
\hline metodológicas activas en sus & Casi siempre & \multicolumn{2}{|r|}{4} & \multicolumn{2}{|r|}{1} & 5 \\
\hline clases. & Siempre & \multicolumn{2}{|r|}{3} & \multicolumn{2}{|r|}{2} & 5 \\
\hline \multicolumn{2}{|l|}{ Total } & \multicolumn{2}{|r|}{9} & \multicolumn{2}{|r|}{3} & 12 \\
\hline \multicolumn{7}{|l|}{ Pruebas de chi-cuadrado } \\
\hline & \multicolumn{3}{|c|}{ Valor } & $\mathrm{Gl}$ & \multicolumn{2}{|c|}{ Sig. asintótica (bilateral) } \\
\hline Chi-cuadrado de Pearson & \multicolumn{3}{|c|}{$1,333^{a}$} & 2 & \multicolumn{2}{|c|}{, 513} \\
\hline Razón de verosimilitudes & \multicolumn{3}{|c|}{1,762} & 2 & \multicolumn{2}{|c|}{,414 } \\
\hline Asociación lineal por lineal & \multicolumn{3}{|c|}{1,222} & 1 & \multicolumn{2}{|c|}{,269 } \\
\hline $\mathrm{N}$ de casos válidos & \multicolumn{3}{|c|}{12} & & & \\
\hline
\end{tabular}

Fuente: Elaboración propia.

Para el análisis de relación de variables se realizaron las siguientes hipótesis:

H0: No existe relación entre la utilización de estrategias metodológica en las clases y la utilización del Aprendizaje basado en problemas como estrategia metodológica en el desarrollo de sus clases.

H1: Existe relación entre la utilización de estrategias metodológica en las clases y la utilización del Aprendizaje basado en problemas como estrategia metodológica en el desarrollo de sus clases. 
Yadira Alexandra Calle-Bermeo; Darwin Gabriel García-Herrera; Sandra Elizabeth Mena-Clerque; Juan Carlos Erazo-Álvarez

En la tabla 2, se puede observar que después de realizar el análisis de relación de variables entre la utilización de estrategias metodológicas activas en clases y el uso del Aprendizaje basado en problemas como estrategia metodológica no se evidenció mayor diferencia ya que en base a los valores obtenidos de significancia bilateral para el Chi cuadrado de Pearson son superiores a 0.05 afirmando que la hipótesis es nula, por lo tanto, no existe relación alguna entre las variables analizadas. Se puede observar que 12 docentes encuestados el $33.33 \%$ utilizan casi siempre estrategias metodológicas activas y un porcentaje menor utilizan siempre el Aprendizaje basado en problemas. Esto demuestra que si bien lo docentes conocen el ABP no lo utilizan de manera constante.

\section{Tabla 3.}

Su profesor aplica en matemática estrategias metodológicas que le hacen razonar * En la asignatura de matemática usted puede fácilmente analizar, interpretar, argumentar y emitir criterios en la solución de un problema planteado.

\begin{tabular}{|c|c|c|c|c|c|}
\hline & & \multicolumn{3}{|c|}{$\begin{array}{c}\text { En la asignatura de matemática usted puede } \\
\text { fácilmente analizar, interpretar, argumentar y } \\
\text { emitir criterios en la solución de un problema } \\
\text { planteado }\end{array}$} & \multirow[b]{2}{*}{ Total } \\
\hline & & A veces & Casi siempre & Siempre & \\
\hline \multirow{3}{*}{$\begin{array}{l}\text { Su profesor aplica en } \\
\text { matemática estrategias } \\
\text { metodológicas que le } \\
\text { hacen razonar }\end{array}$} & A veces & 14 & 6 & 2 & 22 \\
\hline & Casi siempre & 23 & 31 & 10 & 64 \\
\hline & Siempre & 29 & 56 & 72 & 157 \\
\hline \multicolumn{2}{|l|}{ Total } & 66 & 93 & 84 & 243 \\
\hline \multicolumn{2}{|c|}{ Pruebas de chi-cuadrado } & Valor & $\mathrm{Gl}$ & \multicolumn{2}{|c|}{ Sig. asintótica (bilateral) } \\
\hline \multicolumn{2}{|c|}{ Chi-cuadrado de Pearson } & $36,224^{a}$ & 4 & \multicolumn{2}{|c|}{0,000} \\
\hline \multicolumn{2}{|c|}{ Razón de verosimilitudes } & 36,490 & 4 & \multicolumn{2}{|c|}{0,000} \\
\hline \multicolumn{2}{|c|}{ Asociación lineal por lineal } & 31,695 & 1 & \multicolumn{2}{|c|}{0,000} \\
\hline \multicolumn{2}{|c|}{$\mathrm{N}$ de casos válidos } & 243 & & & \\
\hline
\end{tabular}

Fuente: Elaboración propia. 
En la tabla 3, respecto a los estudiantes encuestados se puede observar que el valor de sig. para el Chi cuadrado de bilateral Pearson es menor que 0.05 demostrando que la variable es paramétrica asumiendo que la hipótesis es afirmativa $(\mathrm{H} 1)$, por cuanto existe una relación significativa entre las variables. de acuerdo a la muestra de 243 estudiantes consideran que siempre aplican estrategias metodológicas los docentes permitiendo casi siempre analizar, interpretar argumentar y emitir criterios para un pensamiento lógico, determinándose que esta aplicación no es constante en desarrollar un pensamiento crítico lo que permitirá afianzar de mejor manera la propuesta del ABP., con el trabajo colaborativo para mejores resultados.

Los resultados son similares (Valderrama \& Castaño, 2017), en su investigación solucionando dificultades en el aula: una estrategia usando el aprendizaje basado en problemas en el que se realiza un estudio descriptivo transversal se aplica un cuestionario en escala de Likert en la que sus resultados dan que los estudiantes están de acuerdo en que se utilice las estrategias metodológicas activas para su desarrollo lógico por otro lado los docentes ven la necesidad de utilizar esta estrategia pero necesitan capacitación para tener claro sobre la metodología por lo que no utilizan con eficacia, sabiendo que los estudiantes adquieren conocimientos y competencia por lo que ven la necesidad de capacitarse.

Es importante saber y conocer el manejo de la metodología activa por lo que es necesario utilizar el ABP con el trabajo colaborativo para mejorar el nivel académico del estudiante y llegar a una práctica profesional del docente con estrategias adecuadas para su utilización. 
Yadira Alexandra Calle-Bermeo; Darwin Gabriel García-Herrera; Sandra Elizabeth Mena-Clerque; Juan Carlos Erazo-Álvarez

\section{PROPUESTA}

Luego de los resultados obtenidos en la investigación se puede evidenciar que los estudiantes tienen un nivel aceptable en el razonamiento lógico según encuesta de los docentes, ya que la utilización de estrategias metodológicas son constantes pero la aplicación sobre el Aprendizaje basado en problemas no lo utilizan siempre, por lo que se propone llevar al aula de clases el Aprendizaje basado en problemas (ABP) como estrategia metodológica para la enseñanza en el área de matemática con una alternativa que es incorporar el aprendizaje colaborativo como manejo adecuado para llegar a una mayor comprensión en los problemas lógicos matemáticos.

Los docentes debemos estar conscientes en mejorar la práctica educativa con diversas estrategias metodológicas activas que nos ayude en la enseñanza aprendizaje, buscando la participación activa de los estudiantes para mejorar el razonamiento lógico matemático para fortalecer la práctica del docente y mejorar el aprendizaje del estudiante de esta manera cumplir con los objetivos planteados que dentro del procesos de indagación resuelve preguntas mediante la investigación sobre fenómenos complejos que se le presenten, siendo el centro del aprendizaje el estudiante.

En base a ello mi propuesta es la utilización del ABP., con la estrategia metodológica del trabajo colaborativo, para ello propongo los siguientes pasos a seguir: 
Yadira Alexandra Calle-Bermeo; Darwin Gabriel García-Herrera; Sandra Elizabeth Mena-Clerque; Juan Carlos Erazo-Álvarez

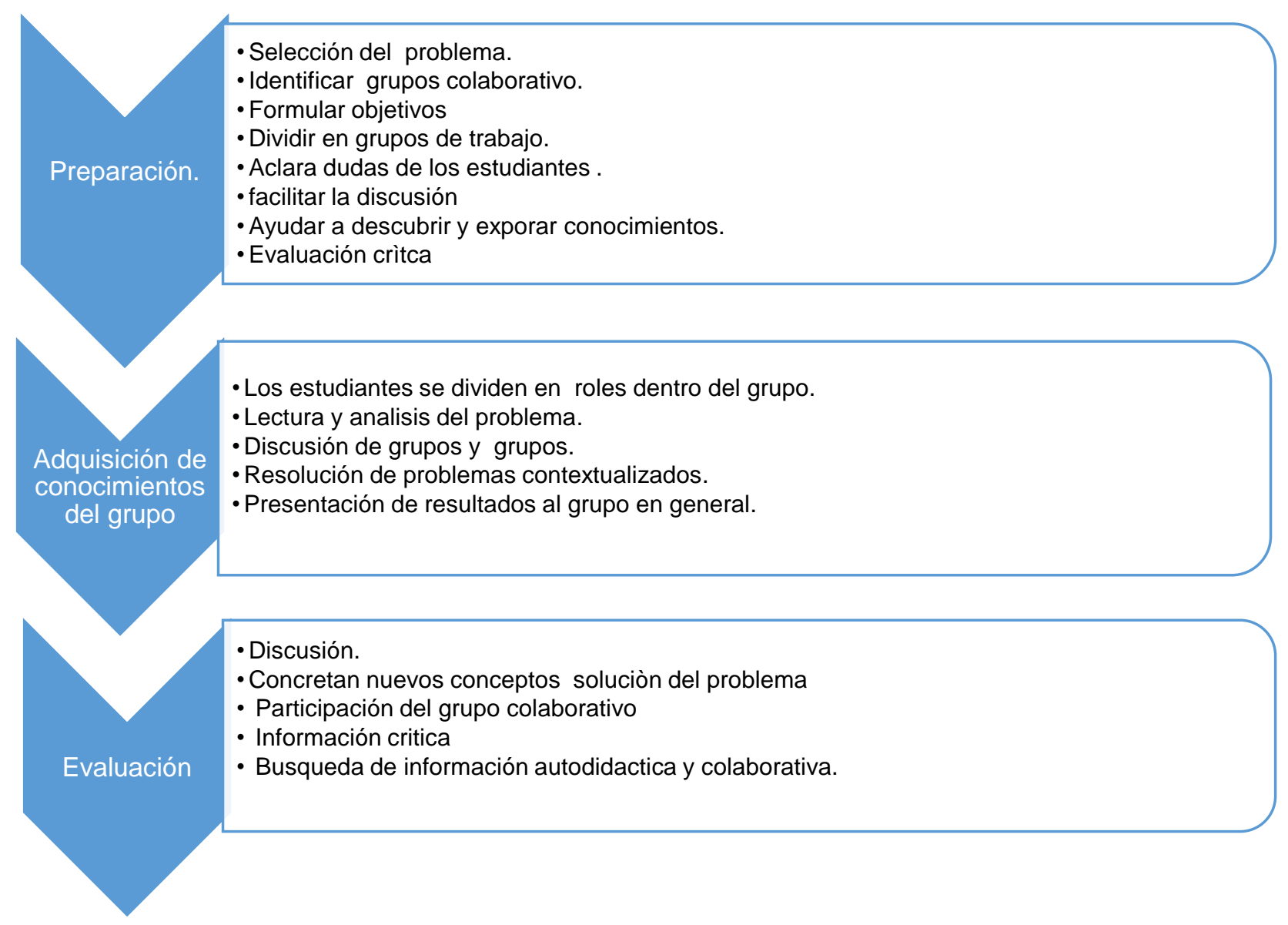

Figura 2. ABP y el trabajo colaborativo una aplicación en el aula. Fuente: Elaboración propia.

Preparación, organización del aula y papel del docente: Se basa en la selección del problema relevante en base al contexto, condicionando el material y espacio necesario para mayor contacto visual tanto docente - estudiante; se identifica con que grupos colaborativos va a trabajar, si es formal abarca el grupo que va desde un periodo de clase a varias semanas, grupos informales se utiliza para trabajar desde unos minutos hasta todo un periodo de clases y grupos base son grupos de largo alcance o de largo plazo pudiendo durar hasta un año lectivo, son grupos heterogéneos, luego se plantea objetivos claros y se divide por grupos de trabajo mediante el juego para mayor comunicación se 
Yadira Alexandra Calle-Bermeo; Darwin Gabriel García-Herrera; Sandra Elizabeth Mena-Clerque; Juan Carlos Erazo-Álvarez

aclara dudas, propone o se forma debates y llegan a acuerdos para promover una evaluación crítica.

Adquisición de conocimientos para la resolución de problemas del grupo: Los estudiantes se dividen en roles para una participación activa en el grupo (supervisor, motivador abogado del diablo, administrador de materiales, secretario, controlador del tiempo entre otros), a continuación analizan y dan lectura del problema matemático planteado llegan a una discusión determinando lo que saben y lo que no saben en pequeños y grandes grupos y sus contraposiciones, el docente es guía para facilitar la información necesaria para que el problema sirva de interés para descubrir el objetivo planteado y puedan llegar a la resolución de problemas con una o varias alternativas a base de un trabajo colaborativo dando oportunidad a que todos los estudiantes sean participativos, respeten sus ideas y articulen sus propios pensamientos en el grupo.

Evaluación: Como propuesta en la evaluación del ABP con la participación del trabajo colaborativo, el docente se fijará en los objetivos planteados y observará el logro de cada uno de ellos y del grupo a través de la discusión y preguntas abiertas, también se evalúa la búsqueda de información para los nuevos conocimientos y la integración de la teoría con la práctica para que sirva la trasferencia de nuevos conocimientos a nuevos problemas planteados así como las habilidades interpersonales, reflexión, de esta manera cumpliendo con una evaluación crítica y constructiva.

El aprendizaje basado en problemas con la ayuda del trabajo colaborativo debe ser aplicado con procesos adecuados, colocados dentro del plan de clase en los tres momentos que son la anticipación, construcción, consolidación, para obtener resultados positivos en la institución de forma colaborativa creado responsabilidades individuales y colectivas desarrollando su criticidad. 


\section{CONCLUSIONES}

En la actualidad la educacion tiene mayor exigencia y continuo cambio, busca mejores alternativas para obtener un desarrollo critico y analitico, utiliza una variedad de técnicas y estrategias que requieren de un aprendizaje activo e innovador sabiendo que el estudiante debe ser el protagonista y centro del aprendizaje.

Es por ello que el (ABP) aprendizaje basado en problemas a través del trabajo colaborativo genera en los estudiantes curiosidad, motivación e interés por aprender dentro de las clases. facilitando la adquisición e interpretación de un problema matemático, siguiendo los pasos adecuados de la metodología con la ayuda del trabajo colaborativo, que sin duda necesita que el docente se involucre en la práctica educativa para asi conseguir resultados positivos en el desarrollo de habilidades y competencias dentro del pensamiento crítico del docente y del estudiante.

\section{FINANCIAMIENTO}

No monetario

\section{AGRADECIMIENTOS}

A la Escuela de Educación Básica "Joel Monroy" ubicada en la ciudad de Cuenca, parroquia Baños, sector Misicata; por apoyar el desarrollo de la investigación.

\section{REFERENCIAS}

Adolphus, T., Alamina, J., \& Aderonmu, T. (2013). The Effects of Collaborative Learning on Problem Solving. IISTE, 4(25), 96.

Bartau, I., \& Azpillaga, V. (Enero de 2017). Metodología de enseñanza en centros eficaces de la comunidad autónoma del país Vasco RIE, 35(1), 93-112. http://dx.doi.org/10.6018/rie.35.1.225141 
Yadira Alexandra Calle-Bermeo; Darwin Gabriel García-Herrera; Sandra Elizabeth Mena-Clerque; Juan Carlos Erazo-Álvarez

Bartau-Rojas, I., Azpillaga-Larrea, V., \& Joaristi-Olariaga, L. M. (2017). Metodología de enseñanza en centros eficaces de la Comunidad Autónoma del País Vasco. [Teaching methodology in effective centers of the autonomous community of the Basque country]. Revista De Investigación Educativa, 35(1), 93-112. https://doi.org/10.6018/rie.35.1.225141

Caballero-Jiménez, F, \& Espínola-Reyna, J. (2016). El rechazo al aprendizaje de las matemàticas a causa de la violencia en el bachillerato tecnológico. [The rejection of learning mathematics due to violence in the technological high school]. Ra Ximhai, 12(3),143-161.

Chagas, P. (2011). Application of project based learning in the discipline of logistics: a case study. IJM\&P, 2 (1), 8-14.

Fernandez, L., \& Fonseca, S. (2016). Aprendizaje basado en problemas: consideraciones para los graduados en medicina famliar y comunitaria en Ecuador. [Problem-Based Learning: Considerations for Graduates of Family and Community Medicine at Ecuado]. MEDISAN, 20(9), 2150-2163.

Gibert , E., \& Ballester , S. (2013). Promoviendo el aprender a aprender matemática en las clases de la educación secundaria básica. [Promoting learning how to learn mathematics in the classes of the basic secondary education]. Atenas, 1, (21), 103118.

Guanipa, M. (2011). Investigación cuantitativa y cualitativa: interdependencia del método. [Quantitative and qualitative research: method interdependence]. REDHECS, $6(11), 1-5$.

Kyeong-Ha, R. (2003). Problem-based Learning in Mathematics. [Aprendizaje basado en problemas en matemáticas]. Recuperado de https://n9.cl//0jxw

Leiva, F. (2016). ABP como estrategia para desarrollar el pensamiento lógico matemático en alumnos de educacion secundaria. [ABP as a strategy to develop logical mathematical thinking in secondary education students]. Sophia, Colección de la Filosofía de la Educación, 21, 209-224.

Lira, R. (2010). Las metodologías activas y el foro presencial: su contribución al desarrollo del pensamiento crítico. [Active methodologies and the face-to-face forum: their contribution to the development of critical thinking]. Revista Electrónica "Actualidades Investigativas en Educación", 10(1), 1-18. 
Yadira Alexandra Calle-Bermeo; Darwin Gabriel García-Herrera; Sandra Elizabeth Mena-Clerque; Juan Carlos Erazo-Álvarez

Matamorros, W. (2018). Propuesta didáctica de Aprendizaje Basado en problemas dirigida al área de matemática $\left(8^{\circ}\right.$ de educación general básica)caso unidad educativa "Sagrada familia". [Didactic Proposal for Problem-Based Learning directed to the area of mathematics (8th grade of basic general education) educational unit case "Sagrada familia"]. Tesis maestría en ciencias de la educación. Pontificia Universidad Católica del Ecuador. http://repositorio.puce.edu.ec/handle/22000/15115

Ministerio de Educación del Ecuador. (2019). Curriculo de los niveles Educación obligatoria subnivel Superior. [Curriculum of the levels Compulsory education sublevel Superior]. Quito: Ministerio de Educaciòn del Ecuador. Recuperado de https://n9.cl/9gov

Moreno-Pinado, W. E., \& Velázquez Tejeda, M. E. (2017). Estrategia didáctica para desarrollar el pensamiento crítico. [Didactic strategy to develop critical thinking]. REICE. Revista Iberoamericana Sobre Calidad, Eficacia Y Cambio En Educación, 15(2), 53-73.

Pérez, Y., \& Ramirez , R. (2011). Estrategias de enseñanza de la resolución de problemas matemàticos. Fundamentos teóricos y metodológicos. [Teaching strategies for solving mathematical problems. Theoretical and methodological foundations]. Revista de Investigación, 35(73), 169-194.

Schleicher, A. (2016). Desafios pasa PISA. [PISA passes challenges]. RELIEVE, 22(1), 1-8. https://doi.org/10.7203/relieve.22.1.8429

Terán-de-Serrentino, M, \& Pachano-Rivera, L. (2005). La investigación-acción en el aula:tendencias y propuestas para laenseñanza de la Matemática en sexto grado. [Action research in the classroom: trends and proposals for the teaching of Mathematics in sixth grade]. Educere, 9(29),171-179.

Travieso-Valdés, D, \& Ortiz-Cárdenas, T. (2018). Aprendizaje basado en problemas y enseñanza por proyectos: alternativas diferentes para enseñar. [Learning Based on Problems and Teaching by Projects: Different Alternatives for Teaching]. Revista Cubana de Educación Superior, 37(1), 124-133. 
EPISTEME KOINONIA

Revista Electrónica de Ciencias de la Educación, Humanidades, Artes y Bellas Artes

Año III. Vol III. N ${ }^{\circ} 1$. Edición Especial. 2020

Hecho el depósito de Ley: FA2018000022

ISSN: 2665-0282

FUNDACIÓN KOINONIA (F.K).

Santa Ana de Coro, Venezuela

Yadira Alexandra Calle-Bermeo; Darwin Gabriel García-Herrera; Sandra Elizabeth Mena-Clerque;

Juan Carlos Erazo-Álvarez

Valderrama-Sanabria, M. L., \& Castaño-Riobueno, G. A. (2017). Solucionando dificultades en el aula: una estrategia usando el aprendizaje basado en problemas. [Solving difficulties in the classroom: a strategy using problem-based learning]. Revista Cuidarte, 8(3), 1907-18. https://doi.org/10.15649/cuidarte.v8i3.456

C2020 por el autor. Este artículo es de acceso abierto y distribuido según los términos y condiciones de la licencia CreativeCommons Atribución-NoComercial-Compartirlgual 4.0 Internacional (CC BY-NC-SA 4.0) (https://creativecommons.org/licenses/by-nc-sa/4.0/). 